\title{
Sleep Mechanism of Base Station Based on Minimum Energy Cost
}

\author{
Dongsheng Han (D), Bing Zheng, and Zhixiong Chen \\ Department of Electrical and Electronic Engineering, North China Electric Power University, Baoding 071003, China \\ Correspondence should be addressed to Dongsheng Han; handongsheng@ncepu.edu.cn
}

Received 31 March 2017; Revised 11 November 2017; Accepted 28 November 2017; Published 29 March 2018

Academic Editor: Jesus Fontecha

Copyright (C) 2018 Dongsheng Han et al. This is an open access article distributed under the Creative Commons Attribution License, which permits unrestricted use, distribution, and reproduction in any medium, provided the original work is properly cited.

\begin{abstract}
Deploying dense network is a promising technique of fifth-generation communication to meet the challenge of soaring demand from explosive growth of mobile users. However, this technique obviously increases the power consumption, thereby indirectly causing heavy financial burden on mobile network operators and $\mathrm{CO}_{2}$ emission, which is considered as a major threat to the environment. In consideration of energy storage device, self-discharge effect, and preventing repeated switch (PRS) mechanism, a comprehensive power management model for wireless communication system in smart grid is investigated in this study from the power consumption and economic cost aspects. Two base sleep mechanisms, namely, energy cost first (ECF) algorithm and power consumption first (PCF) algorithm, are proposed. The ECF algorithm focuses on the minimum cost of system operation by selecting the low-cost energy scheme. Meanwhile, the PCF algorithm considers the minimum power consumption first and then optimizes the cost. Compared with conventional scheme, simulation results show that the two proposed algorithms can decrease the energy cost of communication base system significantly. Setting an energy storage device can further stabilize the energy cost, while the switching frequency can be reduced to a large extent when combined with the PRS mechanism.
\end{abstract}

\section{Introduction}

The proliferation of multimedia infotainment applications and high-speed mobile devices exacerbates the demand for high data rate and consistent quality of service (QoS). The network area throughput needs to be increased nearly 1000fold over the next 10-15 years [1]. As a result, emerging fifth-generation $(5 \mathrm{G})$ wireless communication systems are urgently required. However, high throughput and low power consumption are seemingly contradictory to the $5 \mathrm{G}$ requirements [2]. Deploying dense networks is a promising approach to solve these issues. Dense network employs an ultradense and irregular operator deployment of low-cost and lowpower base stations (BSs) with high density wherein user load is high [3]. The soaring increase in mobile terminals (MTs) and BSs aggravates the power consumption of wireless communication systems. Mobile network operators (MNOs) have reported that the power consumption of BSs contributes to over $70 \%$ of their electricity bill [4]. The power consumption of the information and communication technology industry and the corresponding energy pollution are becoming major societal and economical concerns [5].

The issue can be tackled from two aspects: the communication side mainly including the energy consumption for data processing and the BS side for the operation of BSs. The energy saving of BS system is extremely important considering that the BS side dominates the high consumption. Power control, discontinuous transmission, and antenna adaptation are three main power-saving radio resource management mechanisms to reduce the power consumption at the BS side [6]. Energy efficiency (EE), which is defined as the benefitto-cost ratio, is considered as an optimal index for balancing area spectral efficiency and power consumption [7-10]. In the index, the service quality per area unit (a.u.) is compared with the associated energy consumption, while the expenditure on energy is still ignored.

$$
\begin{aligned}
& \text { EE } \\
& \text { transmit power }+ \text { circuit power per a.u. }\left[\mathrm{J} / \mathrm{symbol} / \mathrm{km}^{2}\right]
\end{aligned}
$$


Given the dynamic characteristics of mobile network, the mobility and daily routine of users provide the network traffic load with space-time varying characteristics. The sleep mechanism, wherein BSs with low traffics during the offpeak period are shut down, can balance the transmitting and circuitry power consumption and thus achieve the purpose of energy saving in the BS system [8, 11-14]. In [11], the effect of sleep mechanism for optimization resource allocation was determined. The results showed that the proposed switchingoff algorithm receives traffic redistribution and offloading of the BSs to be put into sleep mode. Renewable energy generation can harvest energy from environmental sources (e.g., solar and wind) and is thus of low cost, which is generally lower than the cost of generating energy from the grid [15]. Thus, equipping BSs with renewable energy generators is a promising solution. A reasonable power management model, which describes the relationship between energy supply and power consumption of communication system and lays the foundation for further research on energy expenditure, was presented in $[16,17]$. In [18], a united energy management of coordinated multipoint system was proposed to utilize renewable energy fully. The Lagrange dual method was also employed to minimize the system energy cost with an acceptable QoS.

However, these previous works have ignored the influence of energy storage device and the self-discharge effect with no inherent limitation of system construction, during which the reliability is uncertain when power fails [18]. The relationship between power consumption and energy cost in communication system has not been defined, a preliminary idea reflected in the conference paper [19] that we worked on before. Few works have focused on the variation in user connections, and user mobile, which is an important character of mobile network, has been neglected [16, 17]. The flip-flopping between ON/OFF of BSs needs an enormous amount of power in practical communication system, but this topic has not been discussed yet. In consideration of green communication and economic level, we investigate a comprehensive power management model for wireless communication system in smart grid. The model considers weather condition, user mobility, and generation rate of renewable energy to study the influence of energy storage device, self-discharge effect, and preventing repeated switch (PRS) mechanism. Two base sleep mechanisms are proposed to minimize the total energy cost of communication system. Accordingly, the relationship between power consumption and energy cost can be determined by setting energy price reasonably. Considering the power consumption of switching, which means the BSs switch off which are in active state previously and the BSs turn on which are in sleep state previously, the PRS mechanism can limit the switching frequency in a certain range by setting a reasonable switching factor. Such limitation greatly reduces the actual energy waste and hardware loss.

The remainder of the paper is organized as follows. The comprehensive power management model is described in Section 2, and the problem formulation is presented in Section 3. Two base sleep mechanisms are introduced in Section 4. The simulation results are provided in Section 5, and the conclusions of the study are elaborated in Section 6.

The following notations are used throughout the paper. Boldface letters refer to vectors (lowercase) or matrices (uppercase). $v^{\prime}$ and $v^{H}$ denote the transpose and the conjugate transpose of a complex vector $v$. $\|x\|$ and $|x|$ stand for the Euclidean norm and absolute value of a complex vector $x$, respectively. For simplicity, some variables are defined in Nomenclature.

\section{Comprehensive Power Management Model}

2.1. Communication System Model. We consider a coordinated system with joint processing BSs, the number $N$ of which satisfies $N>1$ (Figure 1). Each base is equipped with $M>1$ antennas of transmission power $P_{t}$ and is powered by independent renewable energy. The supply rates of renewable energy vary by BS owing to the difference in renewable energy generators. MTs are equipped with single antenna of received power $P_{r}$, and the number denoted as $K$ is randomly generated in the BS coverage range. For convenience, we denote the set of BSs and that of MTs as $\mathbf{N}=\{1, \ldots, N\}$ and $\mathbf{K}=\{1, \ldots, K\} ; N^{\prime}$ and $\bar{N}^{\prime}$ are the number of active BSs and sleeping BSs, respectively.

A sleep mechanism is applied to turn the redundant BSs into sleep mode and thus reduce energy cost and power consumption simultaneously. We suppose that the system with two active BSs, which are denoted as $\mathrm{BS}_{1}$ and $\mathrm{BS}_{2}$, can meet the user requirements and that the rest of the BSs are kept in the sleep mode. Then, the received signal for MT $k$ is expressed as

$$
y_{k}=h_{k} x_{k}+\sum_{l \neq k} h_{k} x_{1}+\sigma^{2}, \quad k \in \mathbf{K}
$$

where $h_{k} \in C^{1 \times 2 M}$ is the Rayleigh fading channel vector for MT $k, x_{k} \in C^{2 M \times 1}$ is the precoding signal, $\sum_{l \neq k} h_{k} x_{l}$ is the interuser interference, and $\sigma^{2}$ is the background additive white Gaussian noise at MT $k$. Considering large-scale fading and taking two active BSs as an example, denoted as $\mathrm{BS}_{1}$ and $\mathrm{BS}_{2}$, the channel vector for MT $k$ can be expressed as

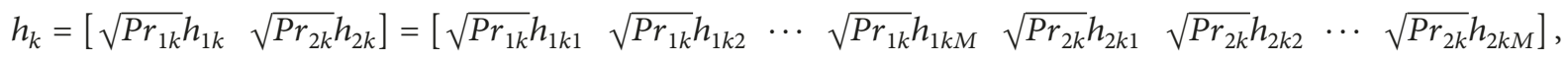

where $\sqrt{\operatorname{Pr}_{1 k}}$ and $\sqrt{\operatorname{Pr}_{2 k}}$ are the received power for MT $k$ from $\mathrm{BS}_{1}$ and $\mathrm{BS}_{2}$.
We assume that $h_{k}, \forall k \in \mathbf{K}$, is available at the transmitter; then, zero-forcing (ZF) precoding can 


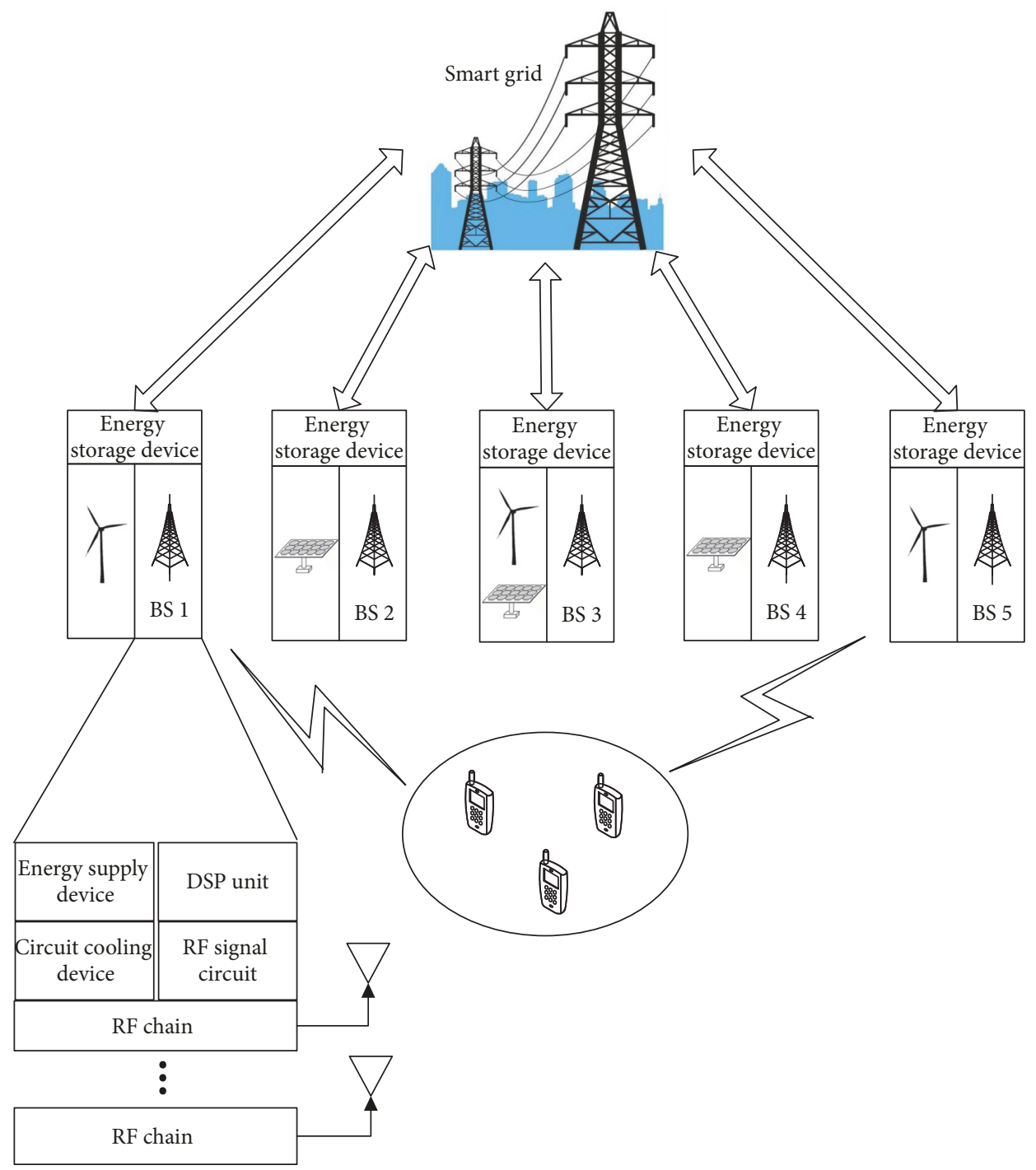

FIGURE 1: Coordinated system of multibase stations with joint processing and BSs of local renewable energy generation.

be used to eliminate the interuser interference; that is,

$$
x_{k}=v_{k} s_{k}
$$

where $v_{k}$ is the precoding vector for MT $k$ and $s_{k}$ is the transmitted signal. Thus, the signal-to-interference-plus noise ratio at $\mathrm{MT} k$ is given by

$$
\operatorname{SINR}_{k}=\frac{\left|h_{k}^{H} v_{k}\right|^{2}}{\sigma^{2}} \text {. }
$$

Given that $N^{\prime}>1$, we use a joint channel vector $h_{k}$ to calculate the precoding vector $v_{k}$, where the channel vector $h_{k}$ is normalized first.

$$
\begin{aligned}
& g_{k}=\frac{h_{k}}{\left\|h_{k}\right\|}, \\
& v_{k}=g_{k}^{\prime} * \operatorname{inv}\left(g_{k} * g_{k}^{\prime}\right) .
\end{aligned}
$$

2.2. Power Consumption Model. Any of the BSs can be powered by smart grid (traditional energy by default), renewable energy (including wind energy alone, solar energy alone, or both), and energy in storage device. The existence of energy storage device can further stabilize the energy cost of BS system and improve the reliability of system. At present, the life of energy storage device can be up to 36 years, with no obvious sign of aging after deep discharge test in the past 4 years. This study ignores the aging phenomenon but focuses on the influence of self-discharge effect.

The generation cycle of new energy at each BS is averaged over $15 \mathrm{~min}$. Thus, each BS possesses 96 time periods over 24 h (i.e., one day), denoted by $\Gamma=\{1, \ldots, 96\}$. The smart grid deploys the peak-valley time-of-use (TOU) tariff strategy. The price of traditional energy varies with time. Notably, $q_{\text {back }}<$ $q_{n, t}$ and $q_{\text {back }}<q_{b}$. We assume that the battery discharge price is fixed. Thus, the cost of self-discharge consumption can be expressed as

$$
S_{t}=C_{t} L c_{b} .
$$




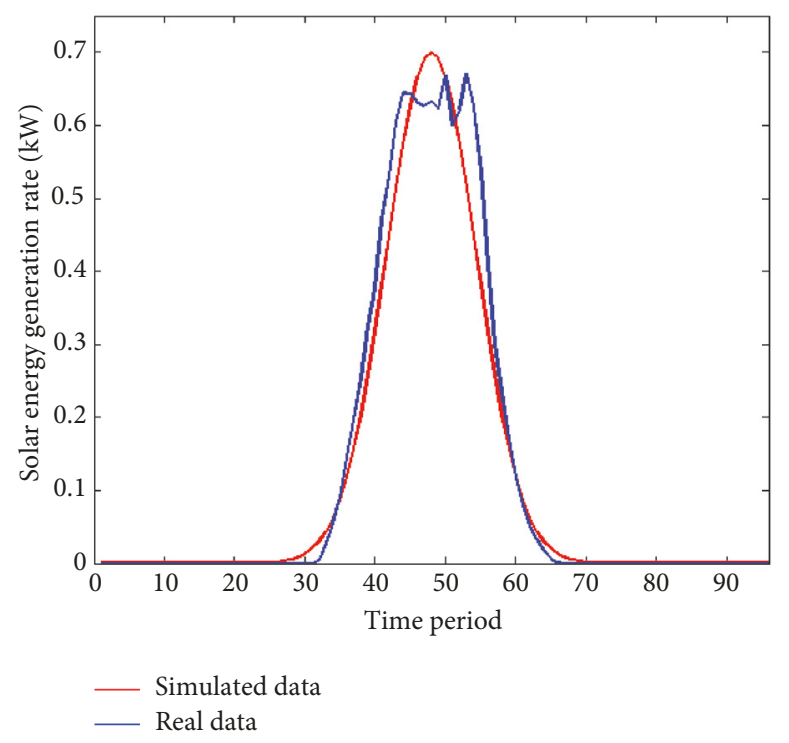

Figure 2: Solar energy generation rate.

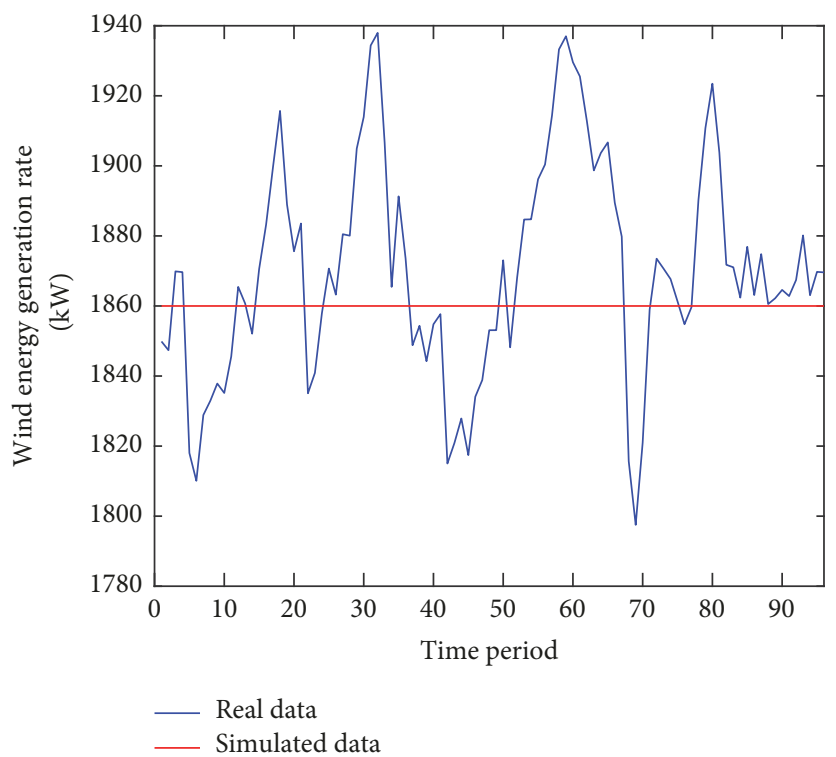

Figure 3: Wind energy generation rate.

The statistical analysis of real data in Figure 2 shows that the solar energy generation rate follows a similar pattern to normal distribution [20]. The rate can be expressed as

$$
r_{1}(t)=\lambda_{s} \exp \left(-\frac{(t-48)^{2}}{81}\right), \quad \lambda_{s} \in\left\{\begin{array}{ll}
\lambda_{s 1} & \lambda_{s 2}
\end{array}\right\}
$$

and the wind generation rate shown in Figure 3 is fixed as

$$
r_{2}(t)=\lambda_{w}, \quad \lambda_{w} \in\left\{\begin{array}{ll}
\lambda_{w 1} & \lambda_{w 2}
\end{array}\right\} .
$$

We use statistical data to indicate the probability of weather conditions and thus determine the different values of $\lambda_{s}$ and $\lambda_{w}$. In accordance with statistics, the probabilities of sunny and cloudy days in a year are denoted as $\left\{P_{\text {sun }} P_{\text {cloud }}\right\}=$ $\left\{\begin{array}{ll}0.6 & 0.4\end{array}\right\}$, and the probabilities of gale and breeze in a year are denoted as $\left\{P_{\text {gale }} P_{\text {breeze }}\right\}=\left\{\begin{array}{ll}0.2 & 0.8\end{array}\right\}$.

In the cellular system, the power consumptions for active BS and sleeping BS are denoted as $P_{\text {acitive }, t}$ and $P_{\text {sleep }, t}$, respectively. The power consumption at each active BS typically includes the transmitting power due to radio frequency (RF) power amplifiers and the fixed power due to other components (e.g., cooling systems, baseband units for data processing, and circuits of RF chains) (Figure 1) [4]. We denote the radiated transmitting power of each antenna for period $t$ by $P_{t x, t} \geqslant 0$ and the fixed power as a constant denoted by $P_{\text {fix }} \geqslant 0$. By combining these powers, we obtain the total 
power consumption for period $t$ at BS $n$, denoted as $P_{\mathrm{BS}, t}$, which can be expressed as

$$
\begin{aligned}
& P_{\mathrm{BS}, t} \\
& \quad= \begin{cases}P_{\text {actice }, t}=P_{\mathrm{fix}}+\eta P_{t x, t}, & \text { if } \mathrm{BS} \text { in active state } \\
P_{\text {sleep }, t}, & \text { if } \mathrm{BS} \text { in sleep state, }\end{cases}
\end{aligned}
$$

where the constant $\eta$ denotes the increment of BS power consumption when transmitting power increases in a unit.

BS switching consumes a large amount of energy in practice. In this study, the switching factor $\delta$ is introduced to reflect the influence of switching. When enabling the BS in sleep mode, the corresponding power consumption is computed by multiplying the switching factor and the original power consumption. If the power consumption is still less than that of the previous BS group, the decision to allow BS to switch on will be executed or the previous group will remain.

\section{Problem Formulation}

Using the proposed model, the resource allocation in energy domain is optimized and the energy cost is minimized under the premise of QoS. Two base sleep mechanisms, namely, energy cost first (ECF) algorithm and power consumption first (PCF) algorithm, are proposed. The ECF algorithm focuses on the minimum cost of system operation by selecting the low-cost energy scheme and determines the active BSs corresponding to the minimum cost for each period. Meanwhile, the PCF algorithm considers the minimum power consumption first and then optimizes the cost, while the active BS group is determined on the basis of the minimum power consumption. The energy source of both algorithms can be renewable energy, traditional energy, or energy in storage device. The best combination of energy needs to be further studied. Notably, the relevant part can be ignored if the BS system is not equipped with energy storage device.

Mathematically, we formulate the two schemes as Scheme 1 and Scheme 2. $P_{r, t}, P_{n, t}$, and $P_{b, t}$ are the electricity supply of renewable energy, traditional energy, and energy in storage device. $P_{e, t}$ denotes the remaining electricity of renewable energy in period $t$. Then, we formulate the energy cost minimizing problem as follows.

\section{Scheme 1 .}

min Price 1

$$
=\min \sum_{n \in N} \sum_{t \in \Gamma}\left(q_{n, t} P_{n, t}+q_{b} P_{b, t}+S_{t}-q_{\mathrm{back}} P_{e, t}\right) .
$$

And Scheme 1 can be decomposed into the energy cost of each time period (Scheme 1-1), which is reformulated as follows.

\section{Scheme 1.1.}

$\min$ price $1_{t}$

$$
=\min \sum_{n \in N}\left(q_{n, t} P_{n, t}+q_{b} P_{b, t}+S_{t}-q_{\mathrm{back}} P_{e, t}\right)
$$

$$
\begin{aligned}
& \text { s.t. } \quad \sum_{n \in N}\left(P_{r, t}+P_{n, t}+P_{b, t}\right) \\
& =\sum_{n \in N^{\prime}} P_{\text {active }, t}+\sum_{n \in \bar{N}^{\prime}} P_{\text {sleep }, t}, \quad \forall t \in \Gamma \\
& P_{e, t}=\left\{\begin{array}{ll}
P_{r, t}-P_{\mathrm{BS}, t}, & \text { if } P_{r, t} \geq P_{\mathrm{BS}} \\
0, & \text { if } P_{r, t}<P_{\mathrm{BS}},
\end{array} \quad \forall t \in \Gamma\right. \\
& P_{r, t} \leq r \times t \\
& P_{t x, t} \leq P_{t x-\max }, \quad \forall t \in \Gamma \\
& \mathrm{SINR}_{k} \geq \mathrm{SINR}_{l}, \quad \forall k \in K \\
& C_{t+1}=C_{t}+R_{b} \times \omega_{t} \text {, } \\
& \text { if } C_{t}<C_{\max }, q_{\text {non }, t}<q_{b} \\
& C_{t+1}=(1-L) C_{t}-P_{b, t} \text {, } \\
& 0 \leq P_{b, t} \leq C_{t} \text {, if } C_{t} \geq 0 .
\end{aligned}
$$

The constraint in (13) ensures that the power input is equal to the power output in period $t$. Equation (14) is used to calculate the remaining renewable energy. The available value of renewable energy for period $t$ can be calculated by (15), which fully utilizes (8) and (9). Equations (16) and (17) denote the transmitting power constraint for period $t$ and the QoS constraint for MT $k$. The charge process of energy storage device is expressed in (18), while the discharge process considering self-discharge effect is in (19).

We formulate the power consumption minimizing problem as follows.

\section{Scheme 2.}

min $P$

$$
=\min \sum_{t \in \Gamma}\left(\sum_{n \in N^{\prime}}\left(\eta P_{t x, t}+P_{\text {fix }}\right)+\sum_{n \in \bar{N}^{\prime}} P_{\text {sleep }}\right) .
$$

The corresponding energy cost can be expressed as

$$
\text { Price } 2=\sum_{n \in N} \sum_{t \in \Gamma}\left(q_{n, t} P_{n, t}+q_{b} P_{b, t}+S_{t}-q_{\mathrm{back}} P_{e, t}\right) \text {. }
$$

Similar to Scheme 1, the above-mentioned problem is decomposed into the subproblems of each time period, which are given by the following.

Scheme 2.1.

$$
\min P_{t}
$$

$$
=\min \left(\sum_{n \in N^{\prime}}\left(\eta P_{t x, t}+P_{\text {fix }}\right)+\sum_{n \in \bar{N}^{\prime}} P_{\text {sleep }}\right)
$$

price $2_{t}=\sum_{n \in N}\left(q_{n, t} P_{n, t}+q_{b} P_{b, t}+S_{t}-q_{\mathrm{back}} P_{e, t}\right)$

s.t. $(13)-(19)$.

By adding them, we obtain the solutions of (20) and (21). 
Specifically, the conventional scheme is obtained when the renewable energy is ignored. In the scheme, the issue of minimizing energy cost is equivalent to that of minimizing power consumption and can be given as

$\min P$

$$
=\min \sum_{t \in \Gamma}\left(\sum_{n \in N^{\prime}}\left(\eta P_{t x, t}+P_{\text {fix }}\right)+\sum_{n \in \bar{N}^{\prime}} P_{\text {sleep }}\right) .
$$

If $q_{\text {low, }}=\min \left(q_{n, t}, q_{b}\right)$, then the corresponding energy cost can be expressed as

Price 3

$$
\begin{array}{r}
=\sum_{t \in \Gamma} q_{\text {low }, t}\left(\sum_{n \in N^{\prime}}\left(\eta P_{t x, t}+P_{\text {fix }}\right)+\sum_{n \in \bar{N}^{\prime}} P_{\text {sleep }}+S_{t}\right) \\
\text { s.t. (16)-(19). }
\end{array}
$$

Obviously, the diversity of energy is ignored when traditional energy adopting peak-valley TOU tariff strategy is treated as the only source of energy. In this study, we fully utilize low-price energy, such as renewable energy combined with traditional energy, to supply the BS system. This application conforms to the dominant trend. Grid repurchases of the remaining renewable energy can further reduce the energy cost.

\section{Base Sleep Mechanism}

Herein, we present two base sleep mechanisms, namely, ECF algorithm against Scheme 1 and PCF algorithm against Scheme 2.

4.1. ECF Algorithm. To minimize the energy cost of BS system, the ECF algorithm decides the corresponding active BSs with minimum energy cost in each time period, while the redundant BSs are set into sleep mode. The ECF algorithm prioritizes the energy of low price to reduce energy cost and system consumption at the same time. Without the loss of generality, assume that the system only has two active BSs. The steps of the ECF algorithm are described as follows.

Step 1 (initializing). User distribution varies in each period owing to the characteristics of user mobility, and the renewable energy generation rate is determined on the basis of the probability of weather conditions shown in (8) and (9).

Step 2 (ZF precoding). ZF precoding is used to eliminate interuser interference.

Step 3 (calculating power consumption). All possible BS groups with two active BSs denoted as $b_{1}$ and $b_{2}$ are considered, and the corresponding power consumption is calculated one by one.
Step 4 (prioritizing low-price energy to supply). All energy prices are listed and the energy of low price is prioritized to supply. Renewable energy is supplied to the BS system first. If $P_{r, t} \geq p\left(b_{1}, b_{2}\right)$, which means that renewable energy can meet power demand, then this system can be supplied only by renewable energy and the grid will repurchase the remaining electricity $P_{e, t}$. Thus, the energy cost can be obtained as

$$
\text { price } 1_{t}^{\prime}\left(b_{1}, b_{2}\right)=S_{t}\left(b_{1}, b_{2}\right)-q_{\mathrm{back}} P_{e, t} .
$$

Otherwise, the algorithm continues to Step 5.

Step 5 (selecting second low-price energy). The price of traditional energy and that of energy in storage device are compared first and then the energy source with low price is chosen:

$$
q_{l}=\min \left(q_{n, t}, q_{b}\right)
$$

If $q_{n, t}=q_{l}$, then traditional energy of low price is selected as the source. The storage device is charged following (18). Otherwise, the system will be supplied by energy in storage device. The energy cost can be given by

$$
\text { price } 1_{t}^{\prime}(b 1, b 2)=q_{l}\left|p(b 1, b 2)-P_{r, t}\right|+S_{t}(b 1, b 2) \text {. }
$$

Step 6 (calculating the remaining power of energy in storage device). Considering the self-discharge effect, the remaining power of energy in storage device can be calculated using (19).

Step 7. Select the minimum energy cost price $1_{t}$ of each period:

$$
\text { price } 1_{t}=\min \left(\text { price } 1_{t}^{\prime}\left(b_{1}, b_{2}\right)\right) \text {. }
$$

Step 8. Determine the BS group in active mode. The BS group corresponding to the minimum energy cost will be determined to be active and is denoted as $\mathrm{BS}_{1}, \mathrm{BS}_{2}$, respectively:

$$
\left(\mathrm{BS}_{1}, \mathrm{BS}_{2}\right)=\text { find }\left(\text { price } 1_{t}^{\prime}\left(b_{1}, b_{2}\right)==\text { price } 1_{t}\right) \text {. }
$$

Step 9. Add the energy cost of each period to the total cost in a day:

$$
\text { price } 1=\sum_{t \in \Gamma} \text { price } 1_{t}
$$

The flowchart of ECF algorithm is shown in Figure 4.

4.2. PCF Algorithm. The PCF algorithm plans to solve the issue in two stages on the basis of the minimum power consumption to minimize the cost. First, the active group is determined on the basis of the minimum power consumption of the BS system, and the redundant BSs are set into sleep mode. Second, low-cost energy is prioritized to supply active BSs and thus minimize the energy cost of the BS system. This algorithm guarantees that the active BS group operates at a low power. However, the differences in renewable energy rate are ignored, which results in the fact that the corresponding energy cost may be larger than that of the ECF algorithm. The steps of the PCF algorithm are described as follows. 


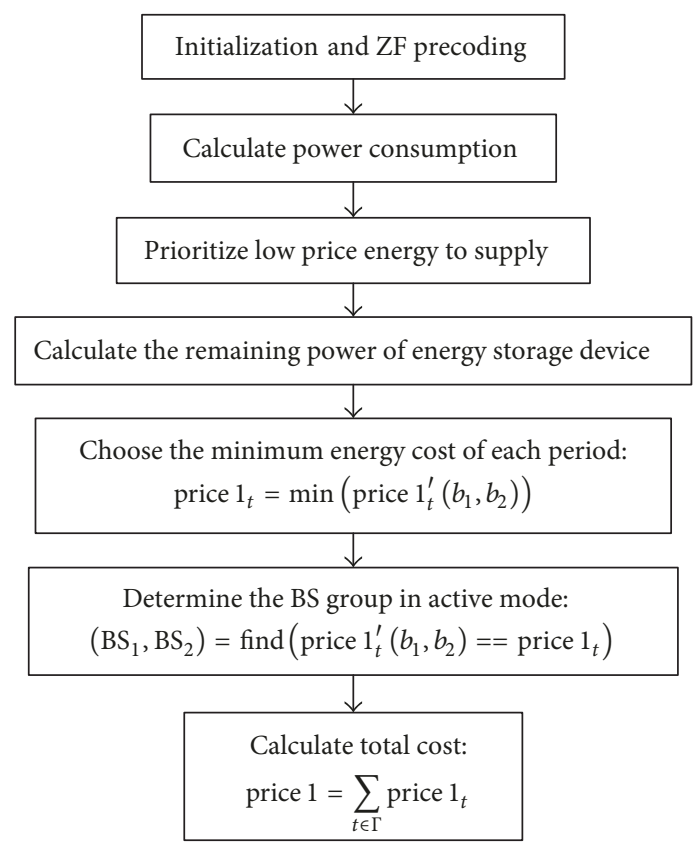

FIgURE 4: The flowchart of ECF algorithm.

Stage 1.

Steps 1-3. These steps are the same as those in the ECF algorithm.

Step 4. Select the minimum power consumption $P_{t}$ of each period:

$$
P_{t}=\min p\left(b_{1}, b_{2}\right) \text {. }
$$

Step 5 (Determining the BS Group in Active Mode). The BS group corresponding to the minimum power consumption will be determined to be active and is denoted as $\mathrm{BS}_{1}^{\prime}, \mathrm{BS}_{2}^{\prime}$ :

$$
\left(\mathrm{BS}_{1}^{\prime}, \mathrm{BS}_{2}^{\prime}\right)=\text { find }\left(p\left(b_{1}, b_{2}\right)==P_{t}\right) \text {. }
$$

Stage 2.

Step 1 (Prioritizing Low-Price Energy to Supply). All energy prices are listed and the energy with low price is prioritized to supply. Renewable energy is supplied to the BS system first. If $P_{r, t} \geq P_{t}$, that is, renewable energy can meet power demand, then this system can be supplied only by renewable energy. The grid will repurchase the remaining electricity $P_{e, t}$. Thus, the energy cost can be obtained as

$$
\text { price } 2_{t}=S_{t}\left(\mathrm{BS}_{1}^{\prime}, \mathrm{BS}_{2}^{\prime}\right)-q_{\mathrm{back}} P_{e, t} .
$$

Otherwise, the algorithm continues with Step 2.

Step 2 (Selecting the Second Low-Price Energy). The price of traditional energy and that of energy in storage device are compared, and the energy source with low price is selected.

$$
q_{l}=\min \left(q_{n, t}, q_{b}\right) .
$$

If $q_{n, t}=q_{l}$, then traditional energy of low price will be selected as the source. The storage device is charged following (18); otherwise, the system will be supplied by energy in storage device first. The energy cost can be given by

$$
\text { price } 2_{t}=q_{l}\left|P_{t}-P_{r, t}\right|+S_{t}\left(\mathrm{BS}_{1}^{\prime}, \mathrm{BS}_{2}^{\prime}\right) \text {. }
$$

Step 3 (Calculating the Remaining Power of Energy Storage Device). Considering the self-discharge effect, the remaining power of energy storage device can be calculated using (19).

Step 4. Add the energy cost of each period to the total cost in a day.

$$
\text { Price } 2=\sum_{t \in \Gamma} \text { price } 2_{t}
$$

The flowchart of ECF algorithm is shown in Figure 5.

\section{Simulation Results}

5.1. Parameter Setting. We consider a practical five-BS wireless communication system (with $N=5$ ), denoted as $n=1,2, \ldots, 5$. Each BS is equipped with transmitting antennas $M=4$. The BS system deploys the pattern of joint processing, and the energy sources can be traditional energy, renewable energy, or energy in storage device. Without loss of generality, we assume that BS1 and BS5 are equipped with wind generators, BS2 and BS4 are with solar generators, and BS3 is with the two types of generators. Different choices of renewable energy mean different rates for supply.

To describe the mobility characteristic of users, we randomly generate users (i.e., $K=8$ ) with single antenna in each time period. Corresponding to the above-mentioned system 


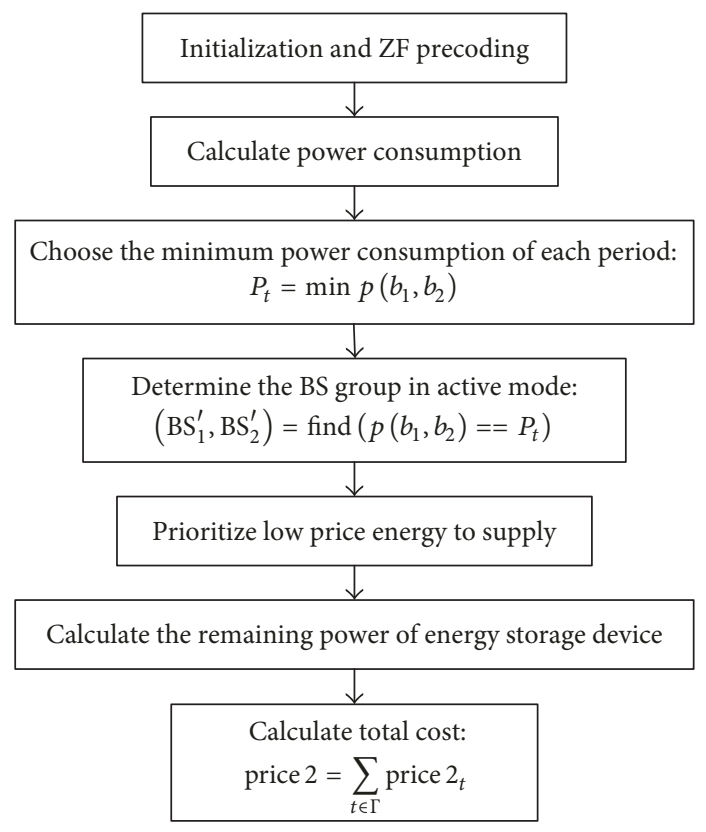

FIgUre 5: The flowchart of PCF algorithm.

TABLE 1: Simulation parameters.

\begin{tabular}{|c|c|}
\hline Parameter & Value \\
\hline The fixed consumption $P_{\text {fix }}$ & $500 \mathrm{~W}$ \\
\hline The power consumption of sleep mode $P_{\text {sleep }}$ & $250 \mathrm{~W}$ \\
\hline Power coefficient $\eta$ & 10 \\
\hline The maximum transmit power $P_{t, \max }$ & $25 \mathrm{~W}$ \\
\hline Noise power $\sigma^{2}$ & $-50 \mathrm{dBm}$ \\
\hline The lowest requirement of SINR $\operatorname{SINR}_{l}$ & $10 \mathrm{~dB}$ \\
\hline$\left\{\begin{array}{ll}\lambda_{s 1} & \lambda_{s 2}\end{array}\right\}$ & $\left\{\begin{array}{ll}0.7 & 0.3\end{array}\right\}$ \\
\hline$\left\{\begin{array}{ll}\lambda_{w 1} & \lambda_{w 2}\end{array}\right\}$ & $\left\{\begin{array}{ll}0.6 & 0.3\end{array}\right\}$ \\
\hline The capacity of energy storage device $C_{\max }$ & $2000 \mathrm{~W}$ \\
\hline Self-discharge rate $L$ & $0.1 \%$ \\
\hline Charge rate of energy storage device $R_{b}$ & $0.1 \mathrm{~W} / \mathrm{s}$ \\
\hline
\end{tabular}

model, we assume that only two BSs are in active mode (i.e., $N^{\prime}=2$ ) and that the other turns to sleep mode to reduce the power consumption and energy cost of BS system. The specific simulation parameters are provided in Table 1.

The traditional energy deploys peak-valley TOU tariff strategy, and the electricity price factor is denoted as $\alpha$. China commercial price instruction shows that 9:00-12:00 and 17:00-22:00 are the peak periods of which the price is $1.33 \alpha / \mathrm{kWh} ; 8: 00-9: 00,12: 00-17: 00$, and 22:00-23:00 are the medium periods of which the price is $0.87 \alpha / \mathrm{kWh} ; 23: 00-8: 00$ of the next day is the valley period of which the price is $0.51 \alpha / \mathrm{kWh}$. The fixed price of energy storage device is $0.6 \alpha / \mathrm{kWh}[18]$.

5.2. Simulation Results and Performance Analyses. Firstly, we investigate the effect of energy storage devices on BSs, which guarantee the reliability of the wireless communication system. An energy storage device with the maximum capacity of $2 \mathrm{~kW}$ is applied on each BS, and the price is fixed to $q_{b}$, which is higher than the lowest price of traditional energy to compensate for the cost of storage hardware. When $q_{b}$ is below the traditional energy price of current moment, the energy storage device can be used as an alternative energy source. When the traditional energy is of low cost, the storage devices do not participate in the energy supply for the BS system and will be connected to a charging source.

Analysis results performed for energy storage device are given in Figure 6. Figure 6(a) illustrates that the averaged price at each time period is observed to vary, following a similar pattern as the corresponding electricity price of traditional energy. The ECF algorithm and the PCF algorithm can reduce the energy cost considerably at each time period regardless of the fact that there are energy storage devices. The ECF algorithm considers the generation rate of renewable energy, the remaining electricity of storage device, and the price of traditional energy and directly optimizes the energy 


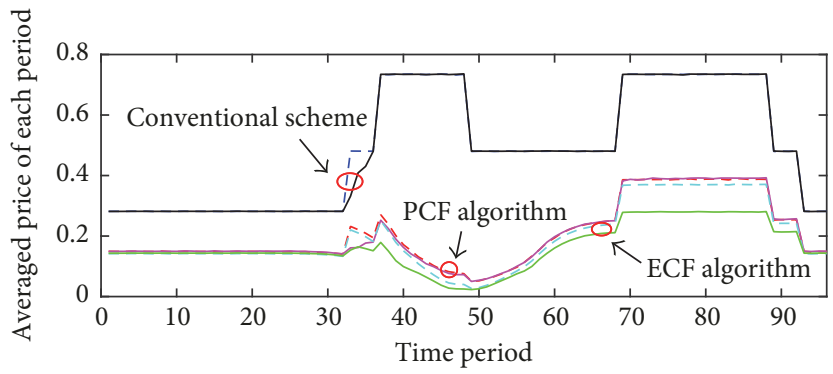

- - Conventional scheme (BS without energy storage device)

- Conventional scheme (BS with energy storage device)

- - - PCF algorithm (BS without energy storage device)

— PCF algorithm (BS with energy storage device)

- - ECF algorithm (BS without energy storage device)

— ECF algorithm (BS with energy storage device)

(a) Comparison of averaged price for energy storage device
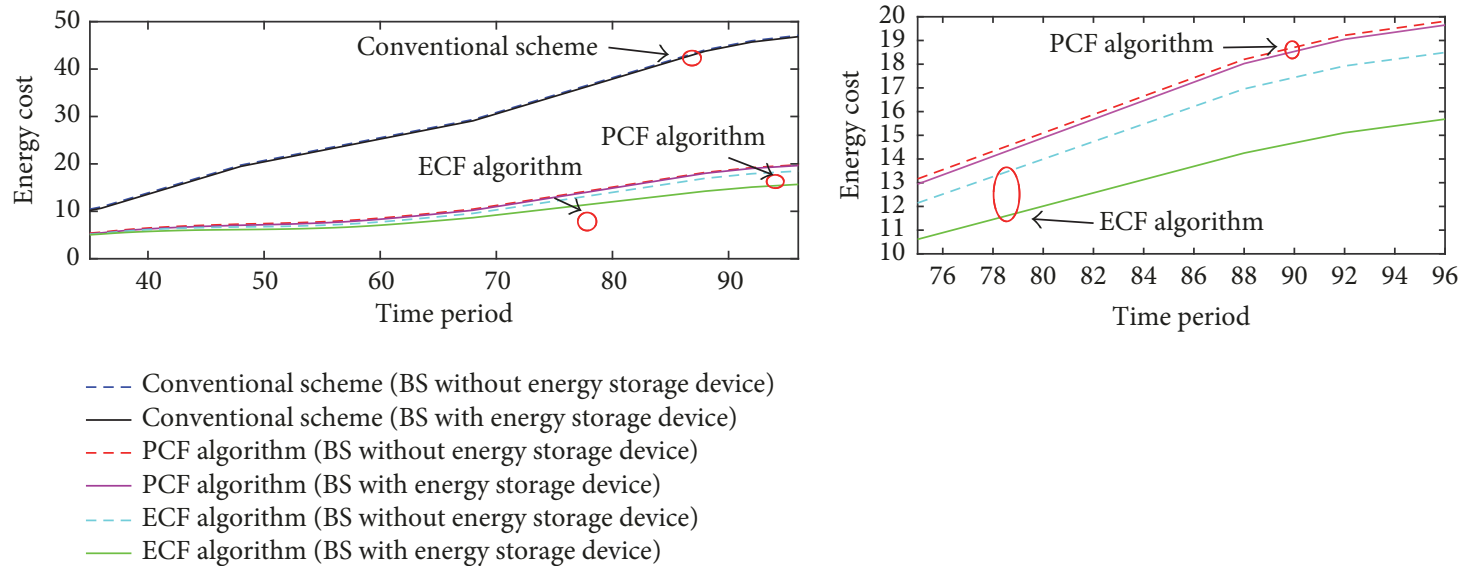

(b) Comparison of total energy costs for energy storage device

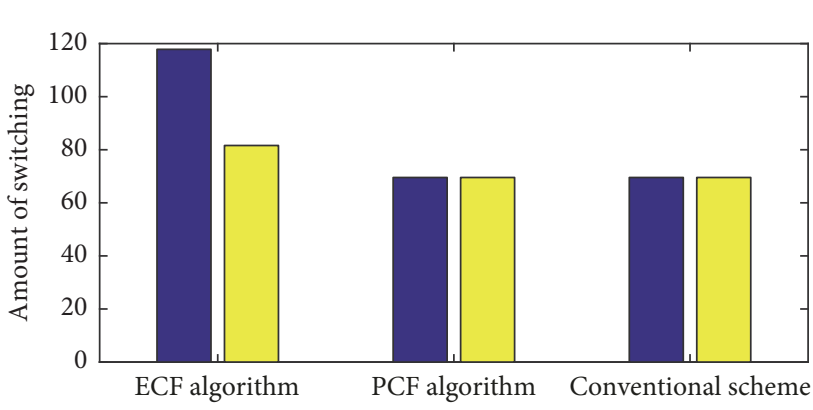

BS with energy storage device $\square$ BS without energy storage device

(c) Comparison of the switching amount for energy storage devices

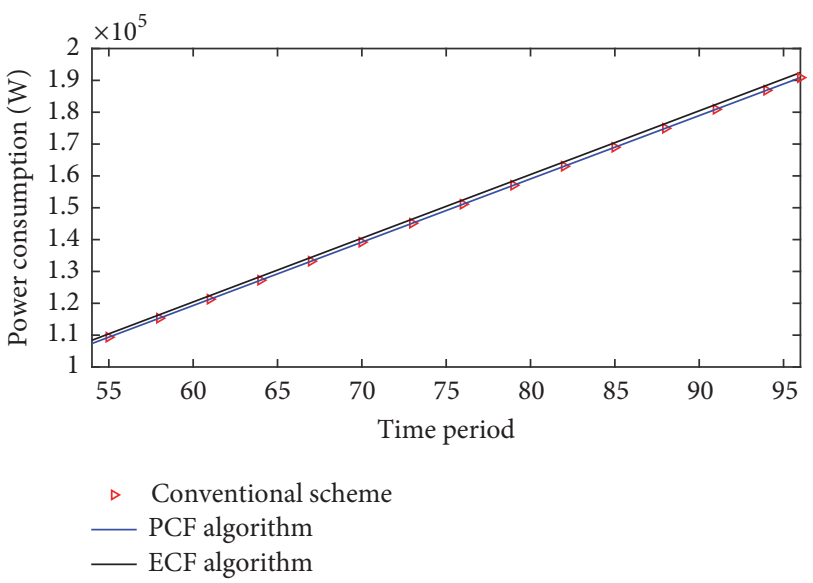

(d) Power consumption for energy storage device

FIGURE 6: Comparisons of (a) averaged price, (b) energy costs, (c) switching amount, and (d) power consumption for energy storage device.

cost of the base system by searching for the lowest one in each time period. The algorithm determines the active BS group, thereby fully utilizing renewable energy although consuming extra energy. The above-mentioned cases use different active BS groups in the corresponding time period. The supply rates of renewable energy are also different, and differences are found among the discharge processes of energy storage device. Thus, the price curves of the ECF and PCF algorithms may overlap or stagger each other. However, ECF algorithm can reduce the energy cost of the BS system to the greatest 


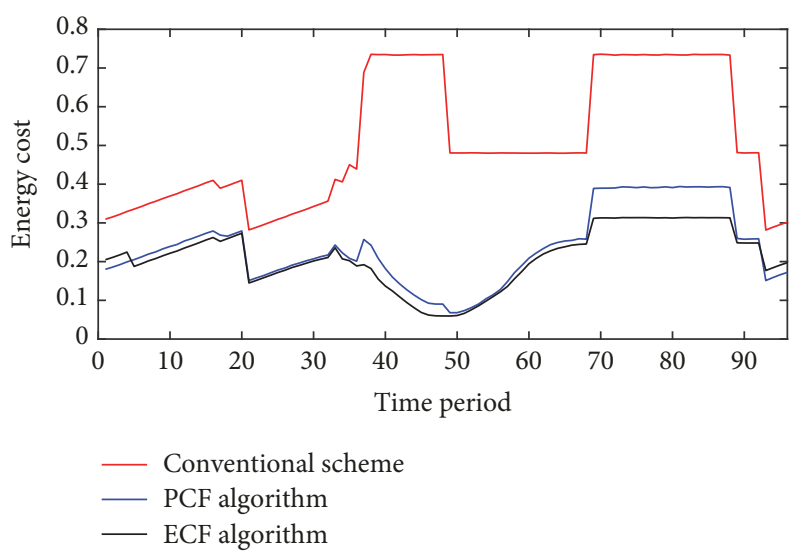

(a) Averaged price considering self-discharge effect

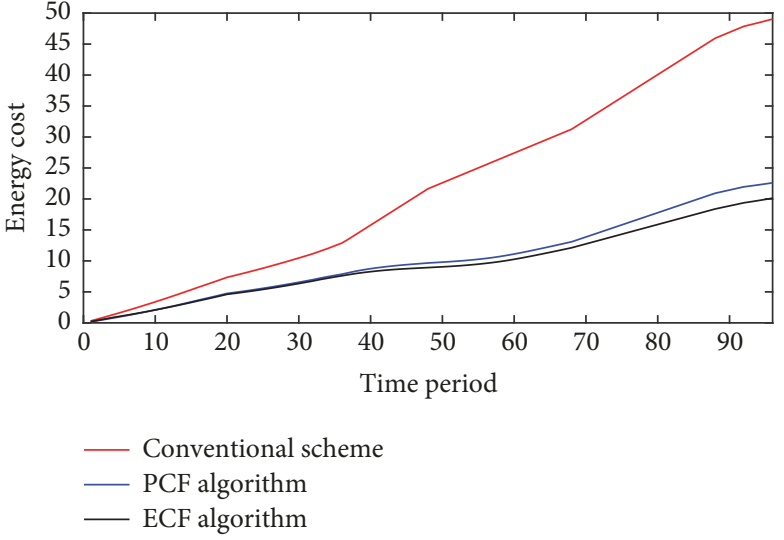

(b) Total energy cost considering self-discharge effect

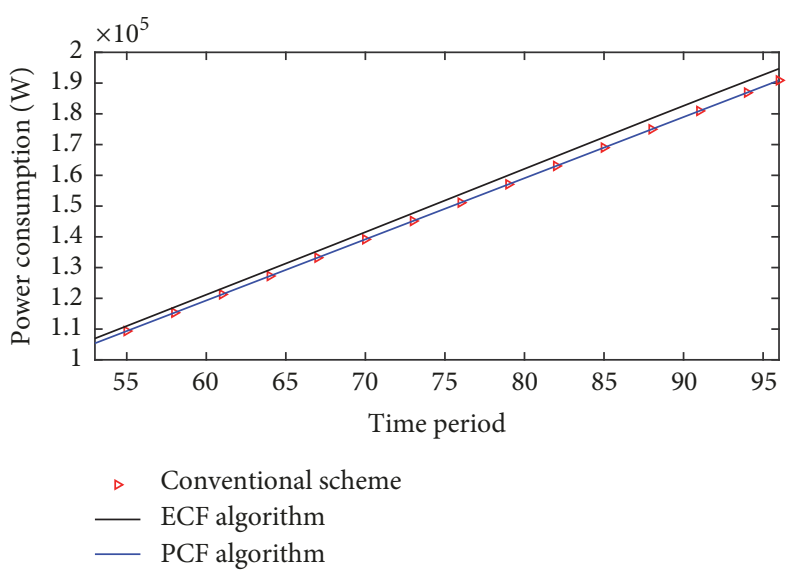

(c) Power consumption considering self-discharge effect

FIGURE 7: Energy cost and power consumption considering self-discharge effect.

extent and achieves a superior performance in general. The total energy cost under different cases without energy storage device is compared (Figure 6(b)). If there are no storage devices, the PCF algorithm achieves $62 \%$ total energy cost reductions over those of conventional scheme, while the ECF algorithm achieves $8 \%$ total energy cost reductions over those of PCF algorithm. At the same time, the existence of energy storage device can further stabilize the price and improve the reliability of the system. The energy costs of all three cases are decreased, the price curves are smooth, and the gap between the two algorithms increases. The ECF algorithm can achieve $68 \%$ energy cost reductions, whereas the PCF algorithm can achieve 58\% reductions. From Figure 6(c), we can observe that the ECF algorithm switches frequently. In other words, this algorithm reduces the energy cost at the expense of switching frequency. Moreover, the switching frequency of the ECF algorithm increases when the energy storage device is switched on, whereas the switching frequencies of other cases remain unchanged. Figure 6(d) describes power consumption under the three cases and it is unrelated to energy storage device. Compared to conventional scheme, it is observed that the power consumption of the PCF algorithm is the same as that of conventional scheme, whereas the ECF algorithm leads to a slight increase of $1851.7 \mathrm{~W}$.

Figures 7(a), 7(b), and 7(c) show the results considering the self-discharge effect of energy storage device. These results confirm that the conclusions are realistic and practical. Figure 7(a) shows that the price curves appear to be bent compared to the case without considering self-discharge effect. Because the resulting averaged price at each time period not only varies with a similar pattern as the corresponding electricity price of traditional energy but also is related to the remaining electricity of energy storage device, the price curve of the ECF algorithm and that of the PCF algorithm will be staggered because of the inconsistent discharge processes under different cases. In general, the two algorithms can greatly reduce the averaged prices. The ECF algorithm, which guarantees the minimum cost of each period, can reduce the energy cost in a large extent and exhibits a superior performance. As shown in Figure 7(b), the total energy costs reach a high level under all three cases. The ECF and PCF algorithms achieve approximately $60 \%$ total energy cost reduction over that of conventional scheme, and the gap between the price curves of the two algorithms is decreased. 


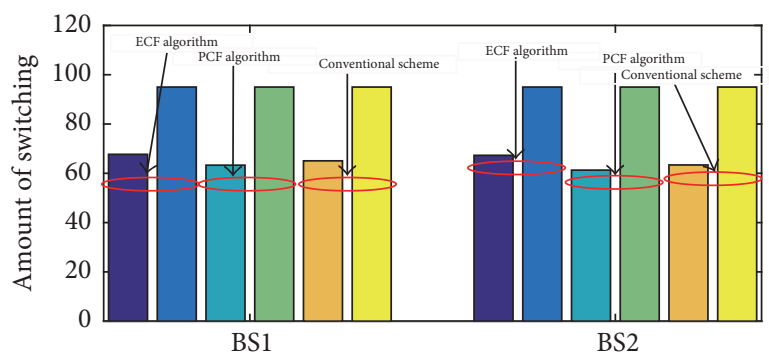

(a) Comparison of switching amount for PRS mechanism

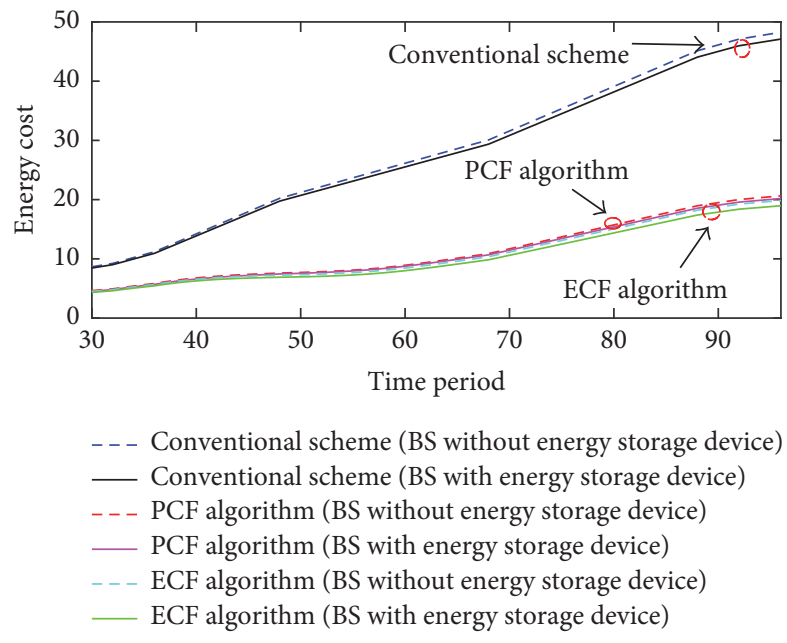

(b) Comparison of total energy costs for PRS mechanism

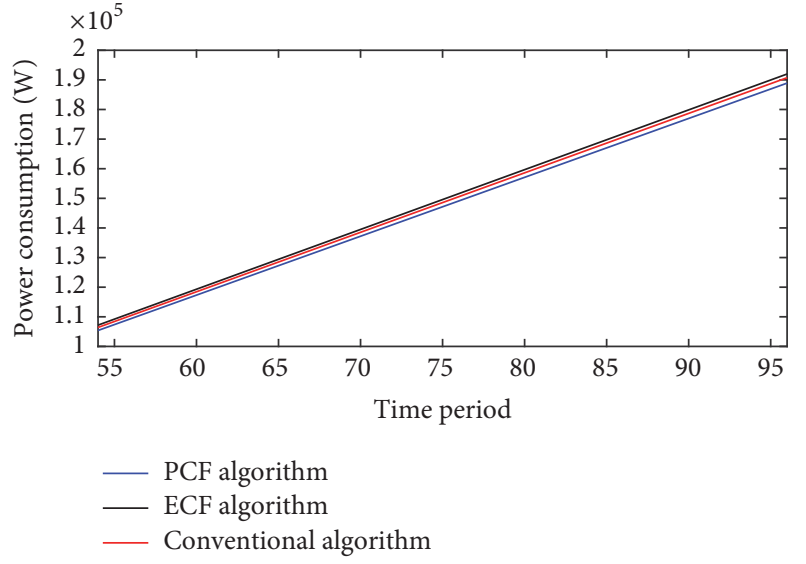

(c) Power consumption for PRS mechanism

FIGURE 8: Comparisons of switching amount, energy cost, and power consumption for PRS mechanism.

Figure 7(c) describes the power consumption of the BS system. It is observed that the power consumption of the PCF algorithm is still the same as that of conventional scheme, while the gap between the ECF algorithm and the two other schemes shows a slight increase of $3703.7 \mathrm{~W}$.

Figures 8(a), 8(b), and 8(c) illustrate the effect of the PRS mechanism, and we set the switching factor as $\delta=1.1$. As shown in Figure 8(a), the switching frequency under each case is reduced significantly after introducing PRS mechanism, and decrement of the PCF algorithm is substantial. On the contrary, the ECF algorithm varies within relatively tight bounds, thereby reducing energy cost at the expense of switching frequency. In Figure 8(b), it is observed that the total energy cost increases under each case. For the PCF algorithm, consider that the switching factor is equivalent to searching for suboptimal active BS group which consumes less energy. For the ECF algorithm, maintaining the previous active group decreases the utilization of renewable energy and the electricity in these storage devices, which is irreconcilable with the goal of minimizing the energy cost. Therefore, the PRS mechanism exerts a serious influence on the ECF algorithm. Figure 8(c) describes the power consumption under three cases after introducing the PRS mechanism. The PCF algorithm still maintains its superiority and consumes the least power compared with that of the two other cases. The ECF algorithm can achieve an optimal effect while consuming a small amount of power, but this algorithm is obviously affected by the switching factor. The ECF algorithm achieves $55.2 \%$ total energy cost reduction over that of conventional scheme and $2.3 \%$ reduction over that of the PCF algorithm.

Figures 9(a) and 9(b) illustrate the power consumption and difference in total energy cost for different switching factors using the ECF algorithm. The influence of switching factor is investigated. For this purpose, the switching factor is changed and the corresponding power consumption is computed (see Figure 9(a)). The gap between the curves narrows nearly to the vanishing point when the switching factor is increased. The differences are obtained by subtracting the original energy cost with $\delta=1$ to the total cost (see Figure 9(b)). With increase in switching factor $\delta$, the energy price increases rapidly, and the price curves become more and more steep from $t=42$ to $t=96$. The gaps between the curves decrease, which is due to the fact that the energy cost for switching on active BS group becomes increasingly high when the switching factor is increased continuously. As a result, the active BS group keeps the previous one.

\section{Conclusion}

In this study, we propose two base sleep mechanisms to reduce the energy cost of wireless BS in a smart grid 


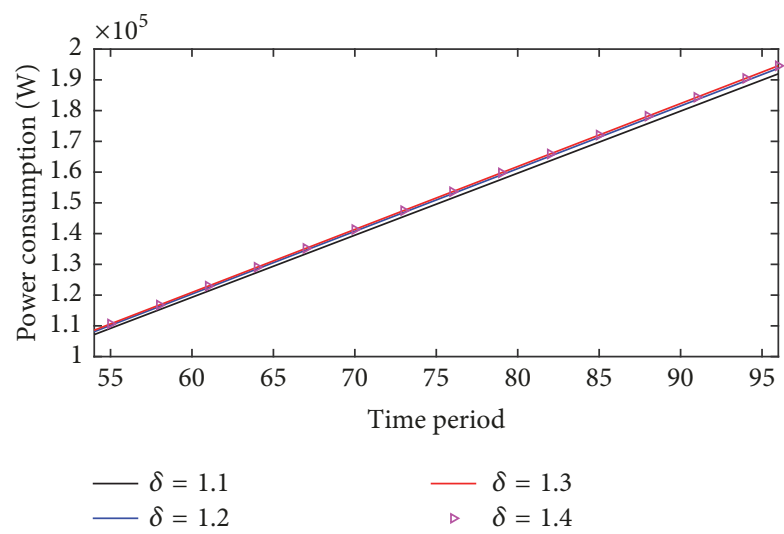

(a) Power consumption for different switching factor

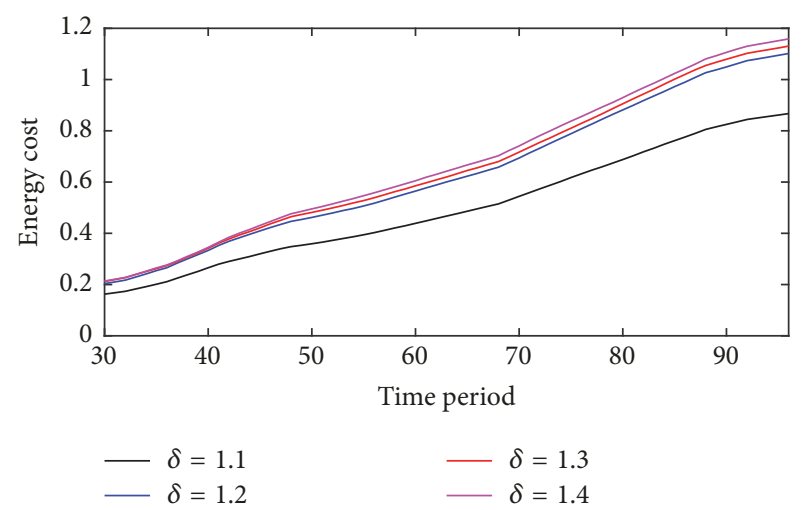

(b) Difference of energy costs for different switching factor

FIGURE 9: Power consumption and energy cost for different switching factor.

environment. We balance the energy cost against the power consumption of the BS system subject to the QoS constraints of each MT, in which the diversity of energy is considered. Each BS can be powered by hybrid sources including renewable and traditional energies. The ECF algorithm achieves significant superiority at the expense of high switching frequency, thereby fully utilizing low-cost energy (i.e., renewable energy and energy in storage device). The PCF algorithm presents a higher energy cost than that of the ECF algorithm but demands minimum power consumption and maintains the switching frequency the same as conventional scheme. Setting an energy storage device can further reduce the energy cost and improve the reliability of the system. Considering the self-discharge effect, we conclude that the ECF and PCF algorithms can achieve considerable energy cost reductions over those of conventional scheme. Meanwhile, the ECF algorithm presents an outstanding performance. The proposed PRS mechanism can decrease the switching frequency significantly and then reduce the actual energy waste and hardware loss caused by the repeated BS switching. Energy cost increases with the increase in switching factor $\delta$ in a certain range, and the system maintains the previous active BS group if the switching factor $\delta$ increases continuously. Accordingly, the performance of the algorithm and the normal operation of the communication system are affected. Thus, the switching factor needs to be set appropriately.

\section{Nomenclature}

$q_{n, t}$ : The electricity price of traditional energy

$q_{\text {back }}:$ The electricity price of grid repurchases of the renewable energy

$q_{b}$ : The electricity price of energy storage device

$C_{t}: \quad$ The available electricity of energy storage device in period $t$

$R_{b}: \quad$ The charge rate of energy storage device

$L: \quad$ The discharge rate of energy storage device

$c_{b}: \quad$ The discharge price of energy storage device $r: \quad$ The renewable production rage

$\mathrm{SINR}_{l}$ : The lowest SINR of user's requirement.

\section{Conflicts of Interest}

The authors declare that they have no conflicts of interest.

\section{Acknowledgments}

This work was supported by the National Natural Science Foundation of China (no. 61771195 and no. 61601182), Natural Science Foundation of Hebei Province (no. F2018502047 and no. F2017502059), and the Fundamental Research Funds for the Central Universities (no. 2018MS091).

\section{References}

[1] Qualcomm, “The 1000x mobile data challenge," Tech. Rep., Qualcomm, 2013.

[2] E. Björnson, E. Jorswieck, M. Debbah, and B. Ottersten, "Multiobjective signal processing optimization: The way to balance conflicting metrics in 5G systems," IEEE Signal Processing Magazine, vol. 31, no. 6, pp. 14-23, 2014.

[3] E. Björnson, L. Sanguinetti, and M. Kountouris, "Deploying dense networks for maximal energy efficiency: small cells meet massive MIMO," IEEE Journal on Selected Areas in Communications, vol. 34, no. 4, pp. 832-847, 2016.

[4] C. Han, T. Harrold, S. Armour et al., "Green radio: radio techniques to enable energy-efficient wireless networks," IEEE Communications Magazine, vol. 49, no. 6, pp. 46-54, 2011.

[5] A. Fehske, G. Fettweis, J. Malmodin, and G. Biczok, "The global footprint of mobile communications: the ecological and economic perspective," IEEE Communications Magazine, vol. 49 , no. 8, pp. 55-62, 2011.

[6] H. Holtkamp, G. Auer, S. Bazzi, and H. Haas, "Minimizing base station power consumption," IEEE Journal on Selected Areas in Communications, vol. 32, no. 2, pp. 297-306, 2013.

[7] Y. Hu, B. Ji, Y. Huang, F. Yu, and L. Yang, "Energy-efficiency resource allocation of very large multi-user MIMO systems," Wireless Networks, vol. 20, no. 6, pp. 1421-1430, 2014. 
[8] K. Son, H. Kim, Y. Yi, and B. Krishnamachari, "Base station operation and user association mechanisms for energy-delay tradeoffs in green cellular networks," IEEE Journal on Selected Areas in Communications, vol. 29, no. 8, pp. 1525-1536, 2011.

[9] S. K. Mohammed, "Impact of transceiver power consumption on the energy efficiency of zero-forcing detector in massive MIMO systems," IEEE Transactions on Communications, vol. 62, no. 11, pp. 3874-3890, 2014.

[10] A. Bousia, E. Kartsakli, A. Antonopoulos, L. Alonso, and C. Verikoukis, "Multiobjective Auction-Based Switching-Off Scheme in Heterogeneous Networks: To Bid or Not to Bid?" IEEE Transactions on Vehicular Technology, vol. 65, no. 11, pp. 9168-9180, 2015.

[11] L. Suárez, L. Nuaymi, D. Grace et al., "A multi-criteria BS switching-off algorithm for $5 \mathrm{G}$ heterogeneous cellular networks with hybrid energy sources," Transactions on Emerging Telecommunications Technologies, vol. 21, no. 7, pp. 923-938, 2016.

[12] S. Han, C. Yang, and A. F. Molisch, "Spectrum and energy efficient cooperative base station doze," IEEE Journal on Selected Areas in Communications, vol. 32, no. 2, pp. 285-296, 2014.

[13] E. Oh and B. Krishnamachari, "Energy savings through dynamic base station switching in cellular wireless access networks," in Proceedings of the Global Telecommunications Conference (GLOBECOM 2010), IEEE, Miami, FL, USA, 2010.

[14] S. Han, C. Yang, G. Wang, and M. Lei, "On the energy efficiency of base station sleeping with multicell cooperative transmission," in Proceedings of the 22nd International Symposium on Personal Indoor and Mobile Radio Communications, PIMRC'11, pp. 1536-1540, IEEE, Toronto, Canada, 2011.

[15] T. Han and N. Ansari, "Powering mobile networks with green energy," IEEE Wireless Communications Magazine, vol. 21, no. 1, pp. 90-96, 2014.

[16] D. Niyato, X. Lu, and P. Wang, "Adaptive power management for wireless base stations in a smart grid environment," IEEE Wireless Communications Magazine, vol. 19, no. 6, pp. 44-51, 2012.

[17] R. Kaewpuang, D. Niyato, and P. Wang, "Decomposition of stochastic power management for wireless base station in smart grid," IEEE Wireless Communications Letters, vol. 1, no. 2, pp. 97-100, 2012.

[18] J. Xu and R. Zhang, "Cooperative energy trading in CoMP systems powered by smart frids," IEEE Transactions on Vehicular Technology, vol. 65, no. 4, pp. 2142-2153, 2014.

[19] D. Han, B. Zheng, and Z. Chen, "A base sleep mechanism for minimizing energy cost," in Proceedings of the 2017 The 7th International Conference on Computer Engineering and Networks, pp. 705-711, Shanghai, China, 2017.

[20] Power Generation, Elia. Brusseis, Belgiu, http://www.elia.be/ en/grid-data/power-generation. 


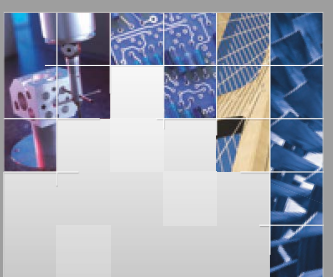

\section{Enfincering}
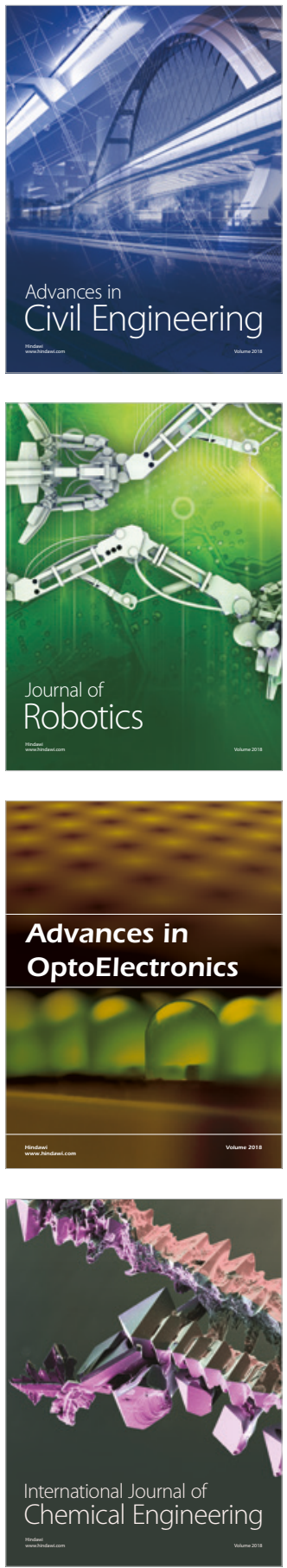

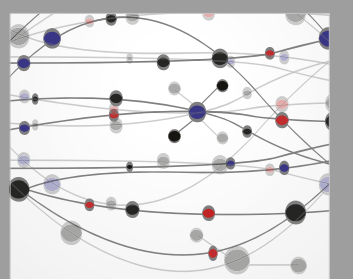

\section{Rotating \\ Machinery}

The Scientific World Journal

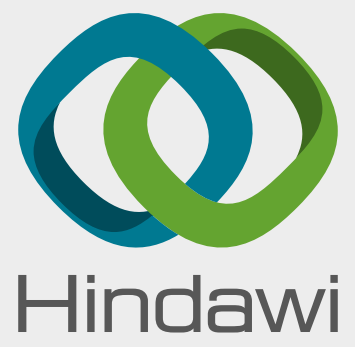

Submit your manuscripts at

www.hindawi.com
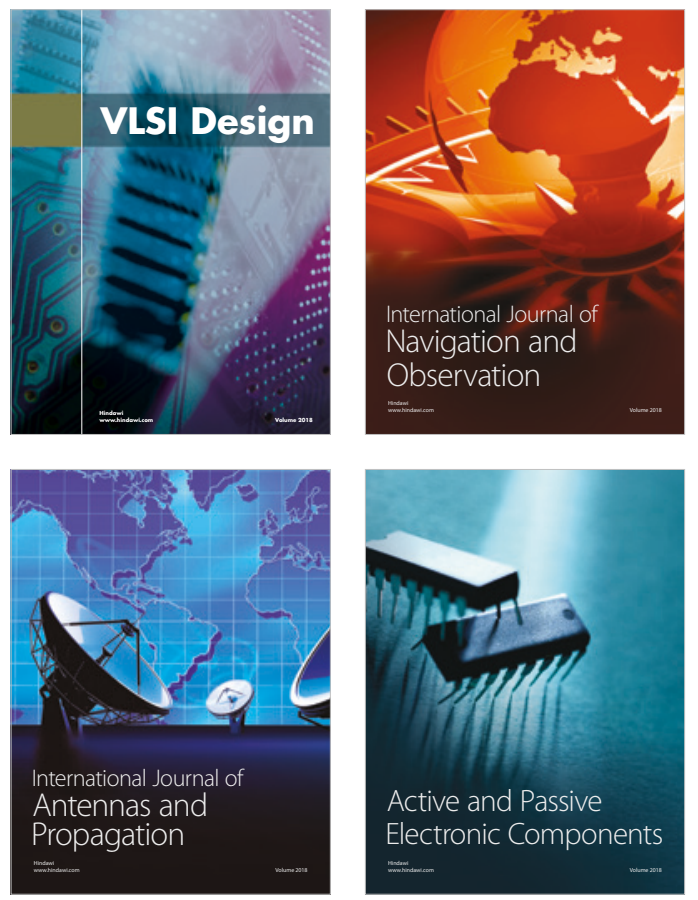
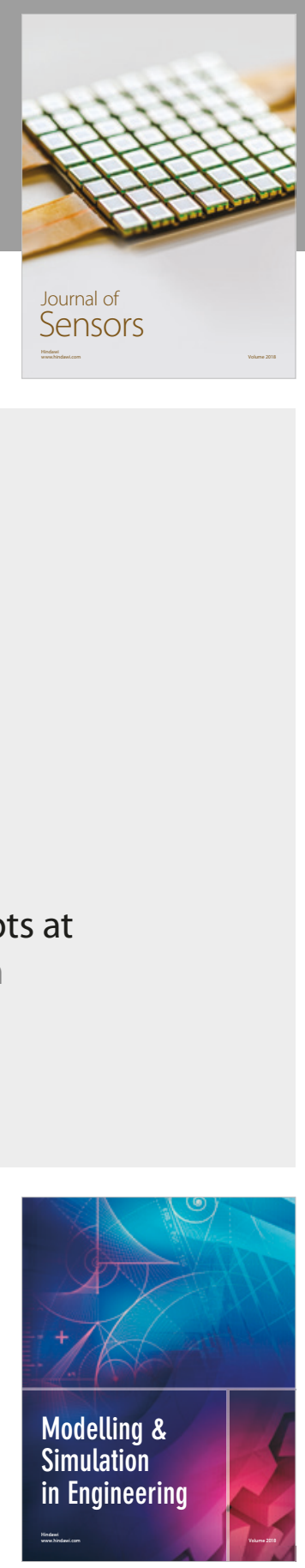

\section{Advances \\ Multimedia}
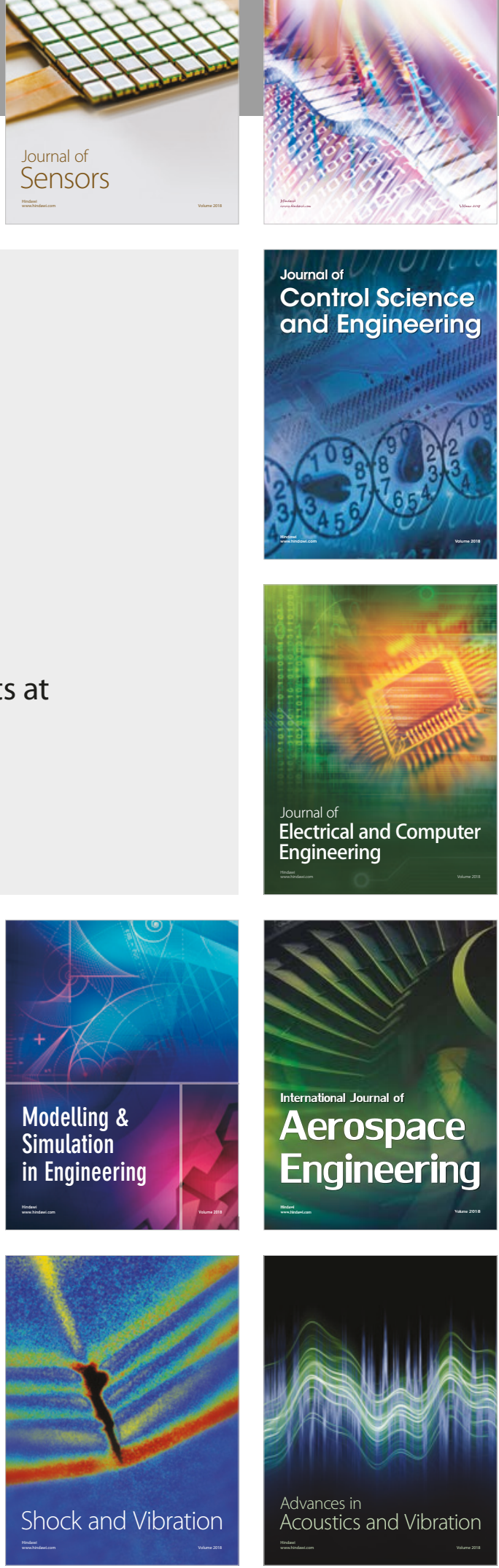